\title{
Immunoreactivity for c-kit and p63 as an adjunct in the diagnosis of adenoid cystic carcinoma of the breast
}

\author{
Mauro G Mastropasqua ${ }^{1}$, Eugenio Maiorano ${ }^{2}$, Giancarlo Pruneri ${ }^{1}$, Enrico Orvieto ${ }^{3}$, \\ Giovanni Mazzarol ${ }^{1}$, Anna R Vento ${ }^{4}$ and Giuseppe Viale ${ }^{1}$ \\ ${ }^{1}$ Division of Pathology and Laboratory Medicine, European Institute of Oncology and University of Milan, \\ School of Medicine, Milan, Italy; ${ }^{2}$ Department of Pathological Anatomy and Genetics, University of Bari, \\ School of Medicine, Bari, Italy; ${ }^{3}$ Division of Pathology, Regional Hospital, Treviso, Italy and ${ }^{4}$ Division of \\ Senology, European Institute of Oncology, Milan, Italy
}

\begin{abstract}
Adenoid cystic carcinoma of the breast represents a unique clinicopathologic entity with a variable histological appearance and a relatively indolent clinical course in most of the cases. Adenoid cystic carcinoma may be difficult to differentiate from infiltrating duct carcinomas, and in particular from tubular and cribriform carcinomas, especially in core or vacuum-assisted biopsies. We evaluated the prevalence of c-kit, p63, and e-cadherin immunoreactivity in a series of 20 adenoid cystic carcinomas, comparing the results with those obtained in a series of infiltrating tubular carcinomas and infiltrating cribriform carcinomas. The hormone receptor status, proliferation labeling index, and HER/2 immunoreactivity had been previously investigated in all the cases. Three (15\%) adenoid cystic carcinomas and all infiltrating tubular and cribriform carcinomas showed estrogen receptor and/or progesterone receptor immunoreactivity $(P<0.00001$ for estrogen and $\boldsymbol{P}=\mathbf{0 . 0 0 0 0 2}$ for progesterone receptors). Adenoid cystic carcinomas consistently lacked any immunoreactivity for HER/2, whereas three (15\%) infiltrating and cribriform carcinomas showed weak and incomplete membrane staining $(P=0.23077)$. Membranous immunoreactivity for c-kit was found in all except one (predominantly basaloid) adenoid cystic carcinomas (95\%), and in none of the infiltrating tubular and cribriform carcinomas $(P<0.00001)$. Nuclear immunoreactivity for $\mathrm{p} 63$ was found in all except three (predominantly basaloid) adenoid cystic carcinomas $(85 \%)$ and in none of the infiltrating tubular and cribriform carcinomas $(P<0.00001)$. All infiltrating tubular and cribriform carcinomas and 18/20 (90\%) adenoid cystic carcinomas showed immunoreactivity for e-cadherin $(P=\mathbf{0 . 4 8 7 1 8})$. In summary, adenoid cystic carcinomas showed the following

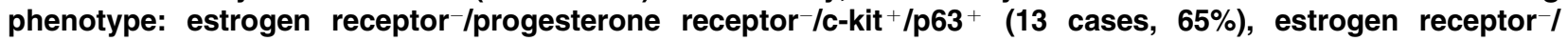
progesterone receptor/c-kit ${ }^{+} / \mathrm{p} / 3^{-}$(three cases, $15 \%$ ), estrogen receptor $-/$ progesterone receptor $^{-} / \mathrm{c}^{-k^{-}} \mathbf{~}^{-} / \mathrm{p} 63^{+}$ (one case, $5 \%$ ), estrogen receptor ${ }^{+} /$progesterone receptor $^{+} / \mathrm{c}_{-} \mathrm{kit}^{+} / \mathrm{p} 3^{+}$(two cases, $10 \%$ ), and estrogen receptor $^{+} /$progesterone receptor $/ \mathrm{c}-\mathrm{kit}^{+} / \mathrm{p} 63^{+}$(one case). By contrast, all the infiltrating tubular and cribriform carcinomas showed the estrogen receptor ${ }^{+} /$progesterone receptor $^{+} / \mathrm{c}-\mathrm{kit}^{-} / \mathbf{p} 63^{-}$phenotype. Our data provide evidence that immunoreactivity for c-kit and/or p63 may be useful in differentiating adenoid cystic carcinomas from other types of breast cancer.
\end{abstract}

Modern Pathology (2005) 18, 1277-1282. doi:10.1038/modpathol.3800423; published online 22 April 2005

Keywords: adenoid cystic carcinoma; breast; c-kit; p63

Adenoid cystic carcinoma is a special type of breast cancer, accounting for $0.1-1 \%$ of all malignant epithelial tumors of the breast. ${ }^{1-3}$ The constituent neoplastic cells are both of luminal and myoepithe-

Correspondence: Professor G Viale, MD, FRCPath, Division of Pathology and Laboratory Medicine, European Institute of Oncology, Via Ripamonti, 435, 20141 Milan, Italy.

E-mail: giuseppe.viale@ieo.it

Received 1 December 2004; revised 17 December 2004; accepted 20 December 2004; published online 22 April 2005 lial type, arranged in a wide spectrum of histological patterns, that is, trabecular, tubular-cribriform, solid, and basaloid, which usually coexist in the same tumor. ${ }^{1-7}$ Most adenoid cystic carcinomas follow a favorable clinical course with a low prevalence of axillary lymph node metastases (approximately $10 \%$ ). Tumors showing a predominant solid pattern of growth however reportedly have a more aggressive behavior, ${ }^{8}$ with distant metastases despite the absence of lymph node involvement and poor 5-year overall survival. ${ }^{6,9-13}$ 
Adenoid cystic carcinoma, especially when showing a predominant tubular-cribriform growth pattern, shares many histological features with infiltrating cribriform and tubular carcinoma. These are more commonly encountered special types of breast cancer, characterized by a long-term survival in more than $80 \%$ of the cases. ${ }^{1,2,7,14}$ The differential diagnosis between adenoid cystic carcinoma, infiltrating cribriform carcinoma, and infiltrating tubular carcinoma on pure morphological grounds may be very challenging, especially when examining small tissue samples obtained by core or vacuum-assisted biopsies. ${ }^{14}$ Assessment of hormone receptor status may be a useful diagnostic adjunct, because most infiltrating cribriform and tubular carcinomas are extensively positive for estrogen and progesterone receptors, whereas adenoid cystic carcinomas are usually negative. Estrogen and/or progesterone receptors immunoreactivity, however, may also be detected in a minor subset of adenoid cystic carcinomas. ${ }^{6,9,15,16}$ More specific and positive markers of adenoid cystic carcinoma would therefore be of diagnostic value.

c-Kit, a transmembrane tyrosine kinase receptor protein (CD117), ${ }^{17}$ and p63, a gene involved in embryonal development and cell differentiation that is constitutively expressed in the basal cell compartment of a variety of epithelial tissues and in myoepithelial cells of the breast, ${ }^{18}$ have been recently proposed as positive markers for adenoid cystic carcinoma. In particular, c-kit and p63 immunoreactivity have been detected in 100 and $87 \%$ of adenoid cystic carcinoma of the salivary glands, respectively. ${ }^{19-21}$ Furthermore, two different groups recently reported, although in abstract form only, that adenoid cystic carcinomas of the breast are also commonly immunoreactive for c-kit, while breast invasive carcinomas not otherwise specified usually lack c-kit expression..$^{22-24}$

e-Cadherin, a transmembrane glycoprotein specifically involved in epithelial cell adhesion, ${ }^{25}$ is a marker of duct carcinomas, including infiltrating tubular and cribriform carcinomas, whereas it is expressed only in a small subset of breast adenoid cystic carcinomas, especially in cases with a predominant tubular or cribriform growth pattern, the solid variants being usually unreactive..$^{5,26}$

To assess the relative value of c-kit, p63, and ecadherin immunoreactivity in the diagnosis of breast adenoid cystic carcinomas, we evaluated immunohistochemically 20 such tumors, comparing the results with those obtained in a cohort of infiltrating tubular and cribriform carcinomas.

\section{Materials and methods}

\section{Patients}

A total of 20 adenoid cystic carcinomas, 10 infiltrating tubular carcinomas, and 10 infiltrating cribriform carcinomas of the breast were retrieved from the files of the Division of Pathology and Laboratory Medicine of the European Institute of Oncology of Milan. Nine adenoid cystic carcinomas cases were sent for consultation with the following original diagnoses: adenoid cystic carcinomas (three cases, 33\%), infiltrating duct carcinoma not otherwise specified (five cases, $56 \%$ ), and infiltrating cribriform carcinoma (one case, 11\%). Representative hematoxylin and eosin-stained sections of all the tumors were reviewed to confirm the tumor type and to assign the differentiation grade according to the recommendations of Ro et al. ${ }^{27}$ Information concerning tumor size and lymph node status were available in all and in 12 cases, respectively. All the cases were restaged using the 2002 TNM classification. ${ }^{28}$ Follow-up data, with a mean duration of 49.4 months, were available for all the patients.

\section{Immunohistochemistry}

All cases (20 adenoid cystic carcinomas, 10 infiltrating tubular carcinomas, and 10 infiltrating cribriform carcinomas) had been immunostained for estrogen receptor, progesterone receptor, Ki-67 antigen, and HER/2 at the time of diagnosis. Consecutive serial sections cut from the same formalin-fixed, paraffin-embedded tissue blocks used for the immunohistochemical evaluation of hormone receptor status, were immunostained for c-kit, p63, and ecadherin using an automated immunostainer (Autostainer, DakoCytomation, Glostrup, Denmark) and a commercially available detection kit (Dako EnVision Plus-HRP), according to the manufacturer's instructions. Clones, sources dilutions, and pretreatments of the antibodies used are listed in Table 1. Built-in positive controls were mast cells (for c-kit), non-neoplastic ductal luminal cells (for c-kit and e-cadherin), and myoepithelial cells (for p63).

In each case, at least 1000 neoplastic cells were evaluated at $\times 400$ magnification, and the percentage of cells showing definite nuclear (for p63), or membranous and/or cytoplasmic (for e-cadherin and c-kit) immunoreactivity was recorded. The

Table 1 Clones, sources, dilutions and pretreatments of the antibodies used

\begin{tabular}{lcccc}
\hline Antibody & Clone & Source & Dilution & Pretreatment \\
\hline Estrogen & 1D5 & DAKO & $1: 100$ & EDTA pH8 \\
Progesterone & 636 & DAKO & $1: 800$ & EDTA pH8 \\
ki67 & MIB-1 & DAKO & $1: 200$ & EDTA pH8 \\
HER/2 & $\mathrm{P}$ & DAKO & $1: 1600$ & EDTA pH8 \\
c-kit & $\mathrm{P}$ & DAKO & $1: 100$ & No \\
p63 & 4A4 & DAKO & $1: 100$ & EDTA pH8 \\
e-Cadherin & 4A2C7 & ZYMED & $1: 400$ & EDTA pH8
\end{tabular}

P: polyclonal. 
Table 2 Clinicopathological and immunohistochemical results in adenoid cystic carcinomas

\begin{tabular}{|c|c|c|c|c|c|c|c|c|c|c|c|c|c|c|}
\hline \# & Age (years) & Site & Stage & $\varnothing(\mathrm{cm})$ & $G$ & $E R$ & $P g R$ & Ki67 & $H E R / 2$ & $G P I \%$ & GPII \% & $c$-kit & p63 & $e-C a d$ \\
\hline 1 & 78 & UEQ & $\mathrm{pT} 1 \mathrm{c} / \mathrm{pN} 1 \mathrm{mi}$ & 1.5 & III & 0 & 0 & 35 & 0 & В 70 & Т 30 & 2 & 0 & 1 \\
\hline 2 & 46 & UEQ & $\mathrm{pT} 1 \mathrm{a} / \mathrm{pNx}$ & 0.2 & I & 0 & 0 & 25 & 0 & C 90 & T 10 & 3 & 3 & 3 \\
\hline 3 & 54 & LIQ & $\mathrm{pT} 2 / \mathrm{pN} 1 \mathrm{a}$ & 2.2 & II & 0 & 0 & 16 & 0 & C 90 & S 10 & 2 & 3 & 3 \\
\hline 4 & 53 & UEQ & $\mathrm{pT} 2 / \mathrm{pNo}$ & 2.2 & III & 0 & 0 & 21 & 0 & S 65 & S 35 & 1 & 3 & 2 \\
\hline 5 & 46 & UEQ & $\mathrm{pT} 2 / \mathrm{pN} 0$ & 2.3 & III & 0 & 0 & 6 & 0 & S 95 & C 5 & 0 & 3 & 2 \\
\hline 6 & 76 & LEQ & $\mathrm{pT} 1 \mathrm{c} / \mathrm{pNx}$ & 1.7 & I & 1 & 1 & 16 & 0 & Т 60 & C 40 & 3 & 3 & 3 \\
\hline 7 & 55 & UIQ & $\mathrm{pT} 1 \mathrm{c} / \mathrm{pN} 0$ & 1.9 & I & 0 & 0 & 18 & 0 & C 95 & T 5 & 2 & 3 & 2 \\
\hline 8 & 51 & LEQ & pT1a/pNo & 0.4 & II & 0 & 0 & 25 & 0 & C 80 & S 20 & 3 & 3 & 2 \\
\hline 9 & 33 & UEQ & $\mathrm{pT} 1 \mathrm{c} / \mathrm{pNx}$ & 1.5 & II & 5 & 3 & 23 & 0 & C 80 & S 20 & 1 & 3 & 1 \\
\hline 10 & 44 & UEQ & $\mathrm{pT} 1 \mathrm{c} / \mathrm{pN} 0$ & 1.7 & I & 0 & 0 & 43 & 0 & Т 80 & C 20 & 3 & 2 & 3 \\
\hline 11 & 70 & UEQ & $\mathrm{pT} 1 \mathrm{c} / \mathrm{pNx}$ & 2.0 & II & 0 & 0 & 38 & 0 & T 80 & S 20 & 1 & 1 & 0 \\
\hline 12 & 46 & UEQ & $\mathrm{pT} 2 / \mathrm{pNx}$ & 2.2 & I & 0 & 0 & 15 & 0 & C 90 & T 10 & 1 & 3 & 3 \\
\hline 13 & 56 & LEQ & pT2/pNo & 2.5 & III & 1 & 0 & 31 & 0 & C 55 & S 45 & 3 & 3 & 3 \\
\hline 14 & 78 & UIQ & $\mathrm{pT} 1 \mathrm{c} / \mathrm{pNx}$ & 2.0 & II & 0 & 0 & 25 & 0 & C 90 & S 10 & 3 & 3 & 1 \\
\hline 15 & 46 & UEQ & $\mathrm{pT} 2 / \mathrm{pNo}$ & 2.5 & I & 0 & 0 & 20 & 0 & C 60 & Т 40 & 3 & 3 & 3 \\
\hline 16 & 53 & LIQ & $\mathrm{pT} 2 / \mathrm{pN} 0$ & 2.6 & III & 0 & 0 & 19 & 0 & S 65 & C 35 & 2 & 3 & 2 \\
\hline 17 & 72 & UIQ & $\mathrm{pT} 1 \mathrm{c} / \mathrm{pNx}$ & 1.2 & II & 0 & 0 & 25 & 0 & $\operatorname{Tr} 75$ & S 25 & 3 & 1 & 3 \\
\hline 18 & 38 & LIQ & $\mathrm{pT} 1 \mathrm{a} / \mathrm{pNx}$ & 0.3 & III & 0 & 0 & 4 & 0 & S 95 & C 5 & 2 & 3 & 2 \\
\hline 19 & 56 & LEQ & $\mathrm{pT} 1 \mathrm{c} / \mathrm{pN} 0 / \mathrm{M} 1$ & 1.6 & III & 0 & 0 & 70 & 0 & B 95 & C 5 & 3 & 0 & 0 \\
\hline 20 & 55 & LIQ & pT2/pNo(sn) & 2.5 & III & 0 & 0 & 50 & 0 & B 75 & Т 25 & 1 & 0 & 1 \\
\hline
\end{tabular}

UEQ: upper external quadrant; LIQ: lower internal quadrant; LEQ: lower external quadrant; UIQ: upper internal quadrant; B: basaloid; T: tubular; C: cribriform; S: solid; Tr: trabecular; GPI: prevalent growth pattern; GPII: secondary growth pattern.

following score system was applied for the immunohistochemical results: 0 , negative; $1+$, up to $25 \% ; 2+, 25-50 \%$; and $3+$, more than $50 \%$ of immunoreactive neoplastic cells. All the slides immunostained for c-kit, p63, and e-cadherin were blindly reviewed by a second investigator, and the interobserver agreement was $98 \%$.

\section{Results}

\section{Clinicopathologic Features of Adenoid Cystic Carcinomas Patients}

All the patients were women, with a mean age of 55.3 years (range 33-78 years). Eleven tumors (55\%) arose in the right and nine (45\%) in the left breast, with a slightly higher prevalence $(60 \%)$ for the upper quadrants (Table 2).

According to the recommendations of Ro et $a{ }^{27}$ six cases $(30 \%)$ were classified as grade I, six as grade II, and eight $(40 \%)$ as grade III. The prevalent $(>50 \%)$ pattern was cribriform in nine $(45 \%)$ cases, solid in four $(20 \%)$, tubular in three $(15 \%)$, basaloid in three $(15 \%)$, and trabecular in the remaining case $(5 \%)$.

A total of $12(60 \%)$ tumors were staged as pT1 (three pT1a and nine pT1c), and eight (40\%) pT2. Of the 12 patients with known lymph node status, 10 $(85 \%)$ were free of metastases, one had a micrometastasis in one lymph node, and one had a (macro)metastasis in one lymph node. One patient with a predominantly basaloid tumor and uninvolved lymph nodes developed lung metastases, which were treated by surgery, 6 months after diagnosis.

\section{Immunohistochemistry}

Three (15\%) adenoid cystic carcinomas showed estrogen or progesterone receptors immunoreactivity, although in a very minor percentage $(1-5 \%)$ of the neoplastic cells. In particular, two cases were immunoreactive for both estrogen and progesterone receptors, and the remaining case for estrogen only (Figure 1). By contrast, all infiltrating tubular and cribriform carcinomas showed immunoreactivity for estrogen receptor and $16(80 \%)$ also for progesterone receptor $(P<0.00001$ for estrogen receptor and $P=0.00002$ for progesterone receptor) in most of the neoplastic cells (ranging from 70 to $95 \%$ ).

Adenoid cystic carcinomas consistently lacked any immunoreactivity for HER/2, whereas three $(15 \%)$ infiltrating tubular and cribriform carcinomas showed weak and incomplete membrane staining $(P=0.23077)$.

The mean value of Ki-67 labeling index was $26 \%$ (range $4-70 \%$ ) in adenoid cystic carcinomas, 10\% (range 4-18\%) in infiltrating cribriform carcinomas, and $8 \%$ (range $4-18 \%$ ) in infiltrating tubular carcinomas.

Membranous immunoreactivity for c-kit was found in all except one (predominantly solid) adenoid cystic carcinomas $(95 \%)$, and in none of the infiltrating tubular and cribriform carcinomas $(P<0.00001)$. Five $(25 \%)$ adenoid cystic carcinomas scored $1+$, five $(25 \%) 2+$, and nine $(45 \%) 3+$. c-Kit immunoreactivity was more prevalent in cribriform, tubular, and trabecular than in solid and basaloid areas (Figure 1).

Nuclear immunoreactivity for p63 was found in all except three (predominantly basaloid) adenoid 

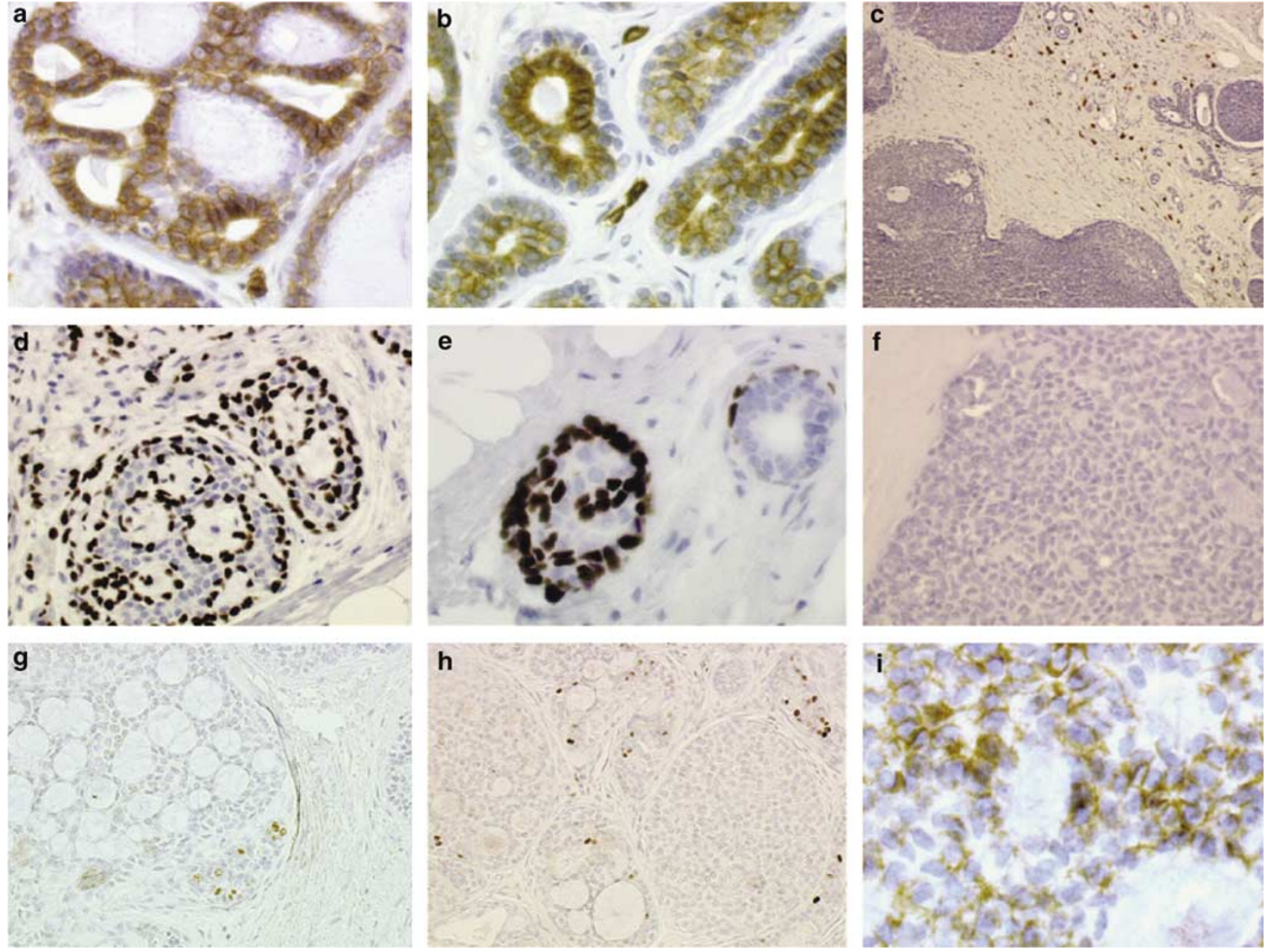

Figure 1 Luminal cells of adenoid cystic carcinoma of the breast show strong membrane immunostaining for c-kit, both in cribriform (a) and tubular (b) growth patterns. On the contrary, solid areas, which are composed by myoepithelial cells are unreactive (c). Myoepithelial cells of adenoid cystic carcinoma show strong nuclear membrane immunostaining for p63, both in cribriform (d) and tubular (e) growth patterns, while they are unreactive in basaloid (f) areas. Adenoid cystic carcinomas occasionally show focal and weak nuclear estrogen (g) and progesterone receptors (h) immunoreactivity, and both, membrane and cytoplasmic, immunoreactivity for e-cadherin (i).

cystic carcinomas (85\%) and in none of the infiltrating tubular and cribriform carcinomas $(P<0.00001)$. Two $(10 \%)$ adenoid cystic carcinomas scored $1+$, one $2+$, and $14(70 \%) 3+$. p63 immunoreactivity was more commonly detectable in cribriform, tubular, trabecular, and solid areas, whereas basaloid areas were usually unreactive (Figure 1).

All infiltrating tubular and cribriform carcinomas and 18/20 (90\%) adenoid cystic carcinomas showed immunoreactivity for e-cadherin $(P=0.48718)$. In particular, in infiltrating tubular and cribriform carcinomas, immunoreactivity was restricted to the cell membrane of most ( $>50 \%$ ) neoplastic cells. In adenoid cystic carcinomas, the pattern of immunostaining was exclusively membranous in seven (35\%) cases, both membranous and cytoplasmic in six $(30 \%)$, and exclusively cytoplasmic in five $(25 \%)$ (Figure 1). With regard to the prevalence of immunoreactive cells, four $(20 \%)$ cases were scored $1+$, six $(25 \%) 2+$, and eight $(40 \%) 3+$.
In summary receptor, adenoid cystic carcinomas showed the estrogen receptor $^{-} /$progesterone re- $^{-}$ ceptor $^{-} / \mathrm{c}-\mathrm{kit}^{+} / \mathrm{p} 63^{+}$phenotype in $13 / 20(65 \%)$ of the cases. In the remaining seven cases, the phenotype was estrogen receptor $^{-} /$progesterone receptor $^{-} / \mathrm{c}_{\mathrm{kit}}{ }^{+} / \mathrm{p} 63^{-}$(three cases), estrogen receptor $^{-} /$progesterone receptor ${ }^{-} / \mathrm{c}_{-} \mathrm{kit}^{-} / \mathrm{p} 63^{+}$(one case), estrogen receptor ${ }^{+} /$progesterone receptor $^{+} / \mathrm{c}^{-}$ $\mathrm{kit}^{+} / \mathrm{p} 3^{+-}$(two cases), and estrogen receptor ${ }^{+} /$ progesterone receptor ${ }^{-} / \mathrm{C}-\mathrm{kit}^{+} / \mathrm{p} 63^{+}$(one case). By contrast, all the infiltrating tubular and cribriform carcinomas showed the estrogen receptor ${ }^{+} /$progesterone receptor ${ }^{+} / \mathrm{c}^{-} \mathrm{kit}^{-} / \mathrm{p} 63^{-}$phenotype.

\section{Discussion}

In the present study, we for the first time document that an immunohistochemical panel including antic-kit and p63 antibodies may be a useful adjunct in 
differentiating adenoid cystic carcinomas from other types of duct cancer.

Adenoid cystic carcinomas of the breast represent a unique clinicopathologic entity characterized by a heterogeneous histological appearance and a relatively indolent clinical course in most of the cases. ${ }^{1-}$ ${ }^{7}$ All the cases of this series showed a coexistence of tubular, cribriform, trabecular, basaloid, and solid growth patterns, and the prevalence of lymph node metastasis was low (approximately 15\%). The solid variant of adenoid cystic carcinomas, however, is reportedly associated with a higher prevalence of distant metastases, ${ }^{6,9,10-13}$ and one pNo tumor of the current series, with a predominant basaloid growth pattern, spread to the lung soon after breast surgery, thus suggesting that a subset of adenoid cystic carcinomas may be characterized by an aggressive behavior.

Given the heterogeneous morphological features, adenoid cystic carcinoma may be difficult to differentiate from infiltrating duct carcinomas, and in particular from infiltrating tubular and cribriform carcinomas, especially in core or vacuum-assisted biopsies. It is worth to note that more than $60 \%$ of the cases which were referred to us for a second opinion had been originally diagnosed as duct carcinomas. It has been previously reported that hormone receptor status may allow to discriminate adenoid cystic carcinomas from duct carcinomas, since it is usually negative in the former and positive in the latter. ${ }^{6,9,15,16}$ Along this line, all infiltrating tubular and cribriform carcinomas analyzed in the present study were consistently immunoreactive for estrogen and progesterone receptors. Nevertheless, in keeping with previous data, ${ }^{6,9,15,16}$ we found that $15 \%$ of adenoid cystic carcinomas also showed estrogen and progesterone receptors immunoreactivity, although in a very minor fraction (up to $5 \%$ ) of neoplastic cells, thus underlying the usefulness of additional and more specific markers.

Our data suggest that c-kit immunoreactivity is the single most reliable diagnostic adjunct in differentiating adenoid cystic carcinomas from infiltrating tubular and cribriform carcinomas: the overwhelming majority (95\%) of adenoid cystic carcinomas and none infiltrating tubular and cribriform carcinomas showed c-kit expression in more than $10 \%$ of neoplastic cells. Two different groups recently reported, although in abstract form only, ${ }^{22-24}$ that all the 33 breast adenoid cystic carcinomas analyzed showed a variable degree of c-kit expression in the ductal luminal component, and that infiltrating duct cancer not otherwise specified were invariably unreactive. The c-kit immunoreactivity is detectable in ductal luminal but not in myoepithelial cells. This may reduce its diagnostic value in cases predominantly composed by solid or basaloid areas, as confirmed by the finding that the only case unreactive for c-kit in our series was predominantly solid. p63 is a highly specific marker of myoepithelial differentiation, that may therefore assist in highlighting the myoepithelial component of adenoid cystic carcinomas. ${ }^{18}$ Accordingly, twothirds of breast adenoid cystic carcinomas (including tumors with prevalent solid features) were immunoreactive for p63 in the present series, a finding in line with previous data in salivary gland tumors. ${ }^{21}$ By contrast, the cases with a basaloid growth pattern lacked any p63 immunoreactivity. In this regard, other authors previously reported that basaloid adenoid cystic carcinomas of the breast are invariably unreactive for the myoepithelial markers smooth-muscle myosin heavy chain and S-100 protein., ${ }^{4,6}$ Whether these tumors should be considered as variants of adenoid cystic carcinomas remains a matter of debate in the absence of positive markers of differentiation. Nevertheless, it is worth to note that in our series basaloid areas frequently coexisted with trabecular, tubular, and/or cribriform areas, which were invariably immunoreactive for the adenoid cystic carcinoma-associated antigens c-kit and/or p63. Taken together, these data corroborate the hypothesis that basaloid areas in adenoid cystic carcinomas may be composed by a multipotent compartment capable of differentiating into both luminal and myoepithelial cells. ${ }^{6,29}$

In keeping with that reported by Shin and Rosen, ${ }^{6}$ our data confirm that immunoreactivity for ecadherin is of little value in differentiating adenoid cystic carcinomas from infiltrating tubular and cribriform carcinomas, since it is shared by most (90\%) of adenoid cystic carcinomas and, as expected, in all infiltrating tubular and cribriform carcinomas. Although most adenoid cystic carcinomas exhibited a cytoplasmic immunostaining pattern, at variance with infiltrating tubular and cribriform carcinomas (where the immunoreactivity was exclusively membranous), this difference is likely to be of little practical value.

\section{References}

1 Rosen PP. Adenoid cystic carcinoma. In: Rosen PP (ed). Rosen's Breast Pathology 2nd edn. Lippincott Williams \& Wilkins: Philadelphia, PA, 2001, pp 535-549.

2 Tavassoli F. Infiltrating carcinoma, special types. In: Tavassoli F (ed). Pathology of the Breast. Appleton \& Lange: Stamford, 1992, pp 403-414.

3 Ellis IO. Adenoid cystic carcinoma. In: Tavassoli F, Devilee P (eds). Tumours of the Breast and Female Genital Organs. Pathology \& Genetics. IARC Press: Lyon, 2003, pp 44-45.

4 Azumi N, Battifora H. The cellular composition of adenoid cystic carcinoma. An immunohistochemical study. Cancer 1987;60:1589-1598.

5 Kasami M, Olson SJ, Simpson JF, et al. Maintenance of polarity and a dual cell population in adenoid cystic carcinoma of the breast: an immunohistochemical study. Histopathology 1998;32:232-238. 
6 Shin S, Rosen PP. Solid variant of mammary adenoid cystic carcinoma with basaloid features: a study of nine cases. Am J Surg Pathol 2002;26:413-420.

7 Page DL. Special types of invasive breast cancer, with clinical implications. Am J Surg Pathol 2003;27: 832-835.

8 Herzberg AJ, Bossen EH, Walter PJ. Adenoid cystic carcinoma of the breast metastatic to the kidney. A clinically symptomatic lesion requiring surgical management. Cancer 1991;68:1015-1020.

9 Arpino G, Clark GM, Mohsin S, et al. Adenoid cystic carcinoma of the breast: molecular markers, treatment, and clinical outcome. Cancer 2000;94:2119-2127.

10 Duan GJ, Liu FX, Yan XC, et al. Clinical significance of E-Cadherin and proliferating cell nuclear antigen expression in adenoid cystic carcinoma [abstract]. Ai Zheng 2002;21:522-525.

11 McClenathan JH, de la Roza G. Adenoid cystic breast cancer. Am J Surg 2002;183:646-649.

12 Foschini MP, Reis Filho JS, Eusebi V, et al. Salivary gland-like tumours of the breast: surgical and molecular pathology. J Clin Pathol 2003;56:497-506.

13 Fukuoka K, Hirokawa M, Shimizu M, et al. Basaloid type adenoid cystic carcinoma of the breast. APMIS 1999;107:762-766.

14 Kontos M, Fentiman IS. Adenoid cystic carcinoma of the breast. Int J Clin Pract 2003;57:669-672.

15 Kleer CG, Oberman HA. Adenoid cystic carcinoma of the breast: value of the histologic grading and proliferative activity. Am J Surg Pathol 1998;22: 569-575.

16 Trendell-Smith NJ, Peston D, Shousha S. Adenoid cystic carcinoma of the breast, a tumor commonly devoid of estrogen receptor and related proteins. Histopathology 1999;35:241-248.

17 Holst VA, Marshall CE, Moskaluk CA, et al. KIT protein expression and analysis of c-kit gene mutation in adenoid cystic carcinoma. Mod Pathol 1999;12: 956-960.

18 Barbareschi M, Pecciarini L, Cangi MG, et al. p63, a p53 homologue, is a selective nuclear marker of myoepithelial cells of the human breast. Am J Surg Pathol 2001;25:1054-1060.

19 Mino M, Pilch BZ, Faquin WC. Expression of KIT (CD117) in neoplasms of the head and neck: an ancillary marker for adenoid cystic carcinoma. Mod Pathol 2003;16:1224-1231.

20 Edwards PC, Bhuiya T, Kelsch RD. Assessment of p63 expression in the salivary gland neoplasms adenoid cystic carcinoma, polymorphous low-grade adenocarcinoma, and basal cell and canalicular adenomas. Oral Surg Oral Med Oral Pathol Oral Radiol Endod 2004;97:613-619.

21 Emanuel P, Wang B, Wu M, et al. p63 Immunohistochemistry in the distinction of adenoid cystic carcinoma from basaloid squamous cell carcinoma. Mod Pathol advance online publication 29 October 2004.

22 Jill P. c-kit expression in adenoid cystic carcinoma of the breast. Pathology 2004;36:362.

23 Crisi GM, Marconi SA, Magari-Judson G, et al. Expression of c-kit in adenoid cystic carcinoma of the breast. Mod Pathol 2004;17(Suppl1):27A (abstract).

24 Matte C, Visscher DW, Reynolds C. c-kit protein expression in adenoid cystic carcinoma of the breast. Mod Pathol 2004;17(Suppl1):41A (abstract).

25 Frixen UH, Behrens J, Sachs $M$, et al. E-cadherinmediated cell-cell adhesion prevents invasiveness of human carcinoma cells. J Cell Biol 1991;113: 173-185.

26 Franchi A, Gallo O, Bocciolini C, et al. Reduced Ecadherin expression correlates with unfavorable prognosis in adenoid cystic carcinoma of salivary glands of the oral cavity. Am J Clin Pathol 1999;111:43-50.

27 Ro JY, Silva EG, Gallager HS. Adenoid cystic carcinoma of the breast. Hum Pathol 1987;18:1276-1281.

28 Sobin LH, Wittekind C (eds). TNM Classification of Malignant Tumours. 6th edn. Wiley-Liss: New York, 2002.

29 Lamovec J, Us-Krasovec M, Zidar A, et al. Adenoid cystic carcinoma of the breast: a histologic, cytologic and immunohistochemical study. Semin Diagn Pathol 1989;6:153-164. 